\title{
Hybrid 240 Ton Off Highway Haul Truck: Quarterly Technical Status Report 18, DOE/AL68080-TSR18
}

This eighteenth quarterly status report for the Hybrid Off Highway Vehicle (OHV) project, DOE Award DE-FC04-02AL68080 presents the project status at the end of March 2007, and covers activities in the eighteenth project quarter, January 2007 - March 2007.

\section{Project Management}

A no-cost continuation has been granted to extend the period of performance until August 31, 2007. The project is on track to complete technical work on or before this date. A project review with Department of Energy staff is being planned for the summer months at Komatsu's Proving Grounds.

\section{Technical Status}

\section{Truck Integration}

All hybrid components have been installed on the Komatsu 830E-AC off-highway truck. A picture of the installation is included at the end of this report showing the two battery modules mounted on the truck. The battery enclosures are mounted to the top deck and front bumper support with vibration isolators to minimize the shock and vibration transmitted to the batteries.

Hybrid operation is controlled from the cab by using a CAN display interface that also shows hybrid status. More advanced parameters of the hybrid system can be controlled through GE's PTU Toolbox running on a laptop in the cab. It is possible to operate the truck in three modes: normal, fuel saving and performance. In normal mode, the hybrid system is disabled and the truck operates as a conventional vehicle. In fuel savings mode, a maximum driver command results in the engine operating at partial power with the hybrid battery pack supplying the remainder. In performance mode, full engine power is supplemented with the hybrid power to provide a higher speed on grade during uphill hauling.

The sodium nickel chloride ZEBRA batteries currently installed on the truck are hot batteries, meaning the internal temperature of the batteries is above $250^{\circ} \mathrm{C}$. During overnight and rest periods, the batteries are plugged in to the charging system. The battery management interface (BMI) installed on each battery properly maintains the internal temperature of the battery by controlling the internal AC heaters. The BMI also communicates to a ground-based battery charger unit to ensure the battery is charged properly and state of charge resets are performed correctly.

While all hardware is installed and operational, further work is required in working through software implementation details and final debugging. Additional truck modification and instrumentation is also being planned to enable higher definition in fuel measurement and other truck performance metrics.

\section{Truck Testing}

Preliminary functional tests have been completed on the hybrid system. Additional instrumentation and improved software is required to enable testing of performance attributes such as fuel savings and productivity improvements.

\section{Static Tests}

The first full-scale on truck testing was static testing. Static tests are performed with the vehicle stationary. The truck engine is started and the alternator is commanded to generate electricity. This power is directed to the battery banks and provides up to a full-power charge. The system can also statically discharge 


\section{DOE/AL68080-TSR18}

battery power to the retarding group, a network of resistors and blower fans normally used for braking (retard) function of the truck.

Both functions of charge and discharge of the battery bank in these static configurations have been tested with good initial results.

\section{Dynamic Tests}

As mentioned above, there are three modes for hybrid truck operation. The "Normal" mode was the first tested. This was an important first step to ensure that the mechanical integrity of the system and to observe relative motion of the battery enclosures to the truck deck through the vibration isolators. During normal driving and heavy braking tests, the enclosures and mounting structures worked well.

Fuel Saving mode and Performance mode have also been tested for functionality. Several dozen trial runs have been performed with good results in terms of functionality of the system. As of this report, there is insufficient data to report on performance impacts to the operating cycle and there is more work to be done on working through software controls to get reliable hybrid performance.

\section{Vehicle modeling}

In parallel with truck testing, the data collected from on-truck testing is being used by the GE Global Research Center to compare previous benefit estimates based on the Matlab/Simulink model developed under this project. Continued testing will enable higher fidelity in the model and help to drive performance improvements.

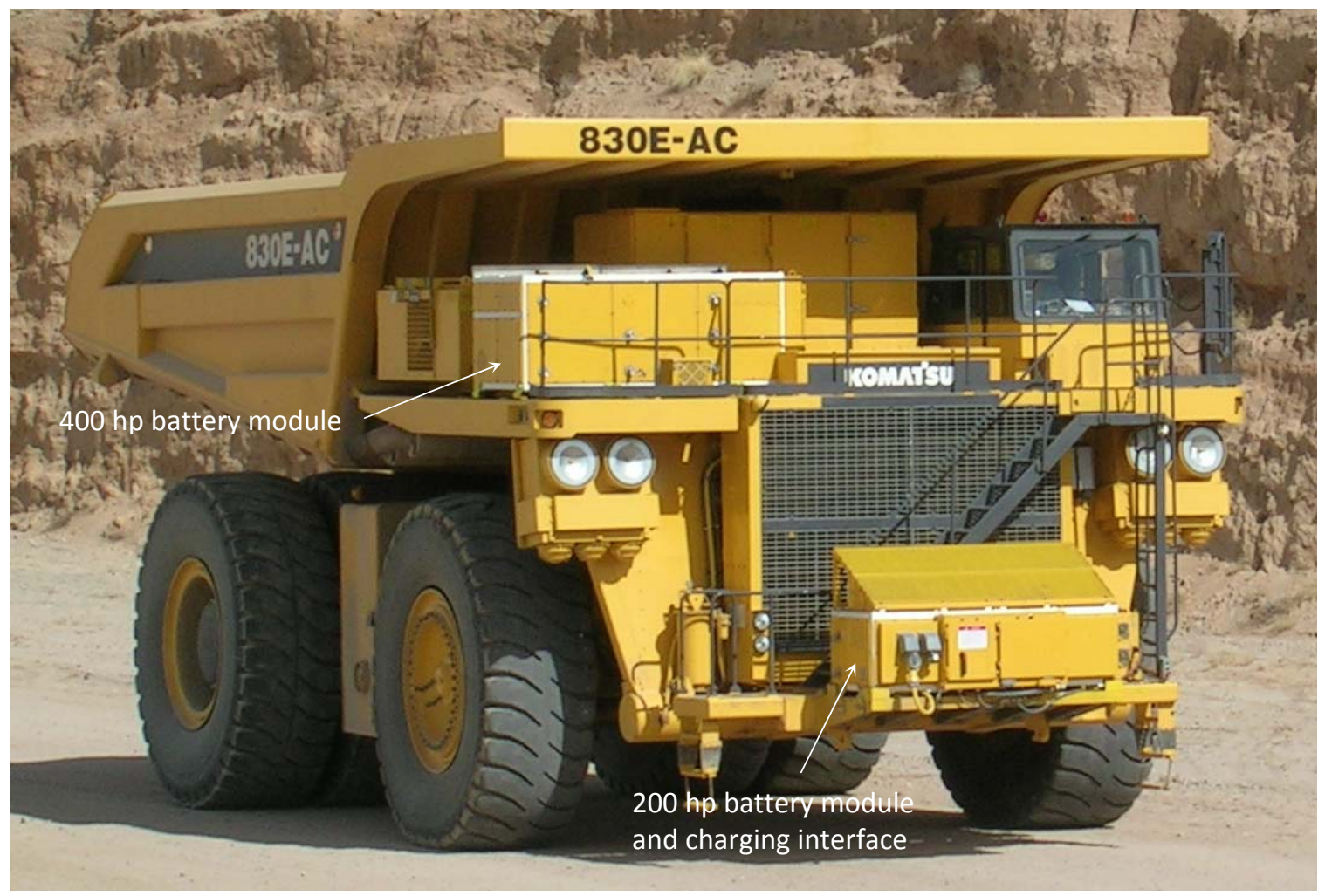

Figure 1 - 240-ton hybrid OHV in test at Komatsu Proving Grounds 